\title{
Microcalorimetry and Thermodynamics
}

\section{Hans-Jürgen Hinz, Regensburg}

(University of Regensburg)

Nearly all chemical reactions in the living cell are catalyzed by specific proteins that have well-defined three-dimensional structures in aqueous solution. Synthesis and degradation occur simultaneously and to prevent destructive interference between them, compartments are created inside cells by lipid molecules (Fig. 1). Lipids ") are highly insoluble in water, but they can form sheetlike bilayer structures that are ideally suited as barriers, due to their unpolar interior and polar exterior (Fig. 1).

Biological systems are subject to the laws of physics and chemistry. They obey the rules of mechanics and of energy conversion just as much as a steam engine does. Therefore thermodynamics provides the appropriate means for characterizing them. Although in principle irreversible thermodynamics might be considered the proper method of describing time dependent processes, in practice, the vast majority of cases can be adequately treated by equilibrium thermodynamics.

Protein Nucleic Acid and Lipid Structures are of Marginal Stability

We live on the edge of stability. This frivolous sounding equivocation is merely to emphasize the lability of biological compounds as compared to the apparent stability of inorganic material. Rocks survive astronomical times, but animals, cells, and their constituents, proteins, nucleic acids, sugars, and lipids undergo a permanent turnover of degradation and novel synthesis. The proper quantity to describe stability of biopolymers is the Gibbs energy, G. At constant temperature and pressure, conditions which usually apply to biological systems, the Gibbs energy change, $\Delta G$, is equal to the maximum net work available for a given change in state. Since $\Delta G$ is a single valued state function, the methods to promote the change in state should in principle be immaterial as long as the initial and final states are clearly specified.

A most convenient way of obtaining the stability parameters is by destruction of the native three- or two-dimensional structures of proteins, nucleic acids, and lipids. This can be achieved chemically by using agents such as guanidinium hydrochloride, urea, or physically, by increasing temperature to unphysiological values, e.g. $100^{\circ} \mathrm{C}$ and higher.

Scanning Microcalorimetry: The Method of Choice to Obtain Model Free Energy Parameters

Use of chemical agents to remove the three-dimensional structure of biopolymers and obtain a random coil state of the macromolecules is possible, because the chemical compounds change the intramacromolecular interactions (hydrogen bonds, electrostatic interactions between permanent charges, dipole-dipole interactions) and/or the interactions between the macromolecule and water (for proteins mainly entropy driven hydrophobic interactions but also hydrogen bonds) by competing for these interactions with the structural units of the macromolecules. Such procedures

\begin{abstract}
b) Schematic diagram of a two-dimensional bilayer structure.

o: hydrophilic moieties on the exterior.
\end{abstract}

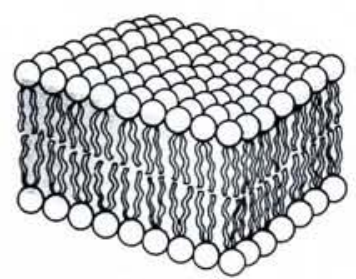

c) Microcalorimetric heat capacity vs. temperature resulting from the bilayer phase transitions indicated below the curve.

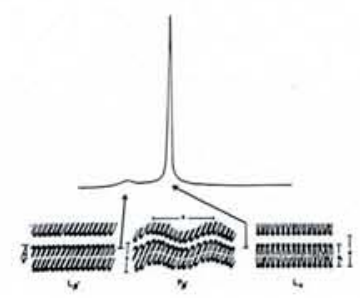

are obviously invasive and require a model of the mode of action of the chemical agents. Therefore the energy parameters derived are only as good as the model on which evaluation is based.

On the other hand, when temperature is used as the perturbing parameter, intrinsic interactions are not altered but are merely overcome by thermal energy. Furthermore as can be seen from the thermodynamic relationship

$$
\Delta G=\Delta H-T \Delta S
$$

a positive entropy change, $\Delta S$, will always favour disruption of a three-dimensional structure, provided the temperature is high enough. Determination of the two thermodynamic parameters $\Delta H$ and $\Delta S$ (the enthalpy and the entropy change respectively) involved in promoting the random coil state of the biopolymers allows us to determine $\Delta G$ unequivocally. The ideal method to obtain these parameters is by high sensitivity scanning microcalorimetry. Conventional calorimetric techniques have always ranked among the most exact physical methods to determine thermodynamic parameters quantitatively. However, it was not until the introduction of highly sensitive, adiabatic, twin scanning microcalorimeters in the last twenty years ${ }^{2,3}$ ) that measurements on biological samples in dilute aqueous solution became possible. The requirement was to determine heat capacity changes as a function of temperature of a $1 \mathrm{mg}$ biological sample over a heat capacity background of $999 \mathrm{mg}$ of water with at least 3-5\% accuracy. Presently available instruments fulfill such requirements.

Fig. 2 shows the sample and reference cell of a DASM-1 Privalov type adiabatic microcalorimeter. The two identical pillshaped gold cells have a volume of $1 \mathrm{ml}$ each. The cells are filled with sample and reference solution via the platinum tubes which at the same time serve as mechanical support. The cells are shielded against heat leakage to and from the surroundings by two so-called adiabatic shields (not shown) which are constantly kept at the same temperature as the cells by automatic heating systems. Applying identical power to sample and reference cell results in identical heating rates of both the cells as long as no endothermic reaction is induced in the sample cell (unfolding of a protein or nucleic acid, conformational changes of lipid phases etc.).

If, however, an endothermic reaction is initiated in the sample cell by temperature rise, the temperature of the sample cell lags behind that of the reference cell. This temperature difference is monito- 


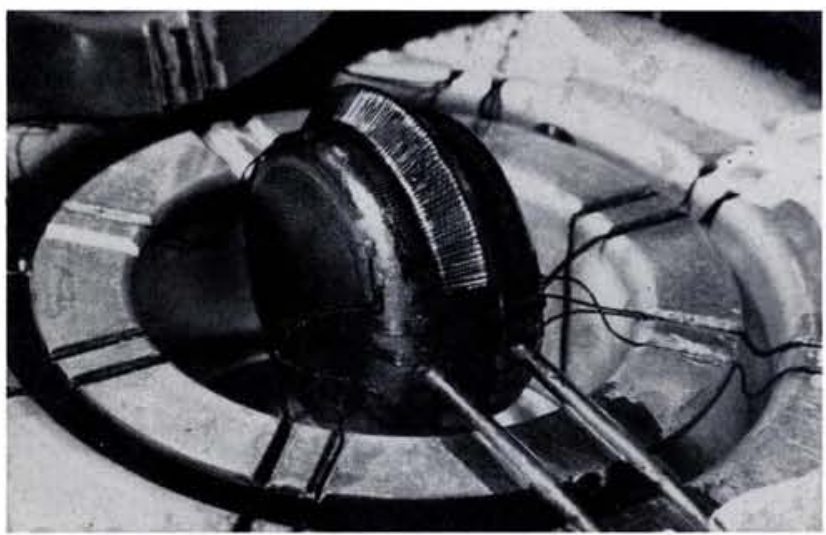

Fig. 2 - Sample and reference cell arrangement of a Privalov type DASM-1 adiabatic scanning microcalorimeter.

red by the thermopile between the cells and is constantly nullified by an automatic feed back system which provides additional power to the sample cell. The extra energy required to maintain a zero temperature difference between sample and reference cell over the temperature range of the measurement is the energy absorbed in the reaction under study.

The equation which the calorimetric determination of the Gibbs energy function $\triangle G(T)$ is based on is:

$$
\begin{aligned}
\Delta G(T)= & \Delta H\left(T_{m}\right)\left[1-T / T_{m}\right]- \\
& \Delta C_{p}\left[T_{m}-T\right]+T \ln T / T_{m}
\end{aligned}
$$
$T$ is the absolute temperature. $\Delta H\left(T_{m}\right)$ is the enthalpy change at the so-called transition temperature $T_{m}$, which is defined as the temperature at which $50 \%$ of the unfolding has occurred (Fig. 3). It can be obtained from the area under the $C_{p}$ vs $T$ curve. $\triangle C_{p}$ is the difference in partial heat capacity between the coiled and the native, structured form of the macromolecule.

\section{Typical Thermodynamic Stability Para- meters of Proteins}

Atomization of one mole of hydrogen molecules, $\mathrm{H}_{2} \rightarrow 2 \mathrm{H}$, requires an enthalpy input of $436 \mathrm{~kJ} / \mathrm{mol}$. This value indicates the order of magnitude of covalent bond energies.

Proteins are mainly stabilized by noncovalent forces, such as $\mathrm{H}^{+}$bonds, charge interactions, dipole forces, and hydrophobic interactions.

To get an idea of the lability of threedimensional structures of proteins we consider $\alpha$-chymotrypsin, a protein of molecular weight 25000 . At $57^{\circ} \mathrm{C}, \mathrm{pH}$ 3.8 , an enthalpy input of $685 \mathrm{~kJ} / \mathrm{mol}$ is sufficient to transform the biologically active macromolecule into an inactive random coil structure.

Another striking feature, typical for the majority of proteins, is the enormous temperature dependence of the unfolding enthalpy, $\Delta H$. In the case of $\alpha$-chymotrypsin, $\Delta H$ varies every degree by approximately $12.6 \mathrm{~kJ} / \mathrm{mol}$, becoming more positive with increasing tempera- ture. Such a behaviour is completely different from that found in gas phase reactions where typically both $\triangle H$ and $\triangle S$ do not change by more than $1-3 \%$ over a temperature range of $100 \mathrm{deg}$.

The Gibbs energy change for unfolding $\alpha$-chymotrypsin at $25^{\circ} \mathrm{C}$ is only $50 \mathrm{~kJ} / \mathrm{mol}$. Since the protein consists of 243 amino acids the average contribution to stability of one structural unit is only $205 \mathrm{~J}$, i.e. one order of magnitude below the thermal energy, RT. So, why is it possible that proteins form well defined three-dimensional structures in aqueous solution at all? There is certainly no clear answer to that question yet, except the somewhat unsatisfactory one, that $\Delta G$ is negative for structure formation at physiological temperatures. However, the very fact that proteins assume unique structures in aqueous solution illustrates another important characteristic of biological systems. Structures are formed in a cooperative manner, and furthermore, the stability of native proteins, as reflected by $\Delta G$, is not proportional to the molecular weight, $M$. For most of the proteins studied so far $(M \cong 6000$ to 100000 ), $\triangle G$ per mol of protein varies by less than a factor of four whereas the number of amino acids varies by a factor of almost 20. This result suggests that Nature does not select these proteins for optimum stability but for a balanced stability-mobility relationship that appears to be essential for catalytic function.

\section{Scanning Microcalorimetry Can Bridge} the Macroscopic and Microscopic

Heat capacity measurements as a function of temperature not only permit us to calculate the most accurate thermodynamic stability parameters of biological systems, they also make it possible to gain information on the path of unfolding by a shape analysis of the transition curves ${ }^{4,5}$ ). In the course of unfolding a biological macromolecule by temperature increase, various enthalpy states will be occupied. Distribution over these enthalpy states is of the Boltzmann type, and the probability, $P_{i}$, of each macrostate is given by:

$$
P_{i}=\exp \left(-\Delta G_{i} / R T\right) / Q
$$

where $\Delta G_{i}$ is the Gibbs energy difference between the partially unfolded structure and the intact macromolecule, and $Q$ is the partition function, $\Sigma$ exp $\left(-\triangle G_{i} / R T\right)$. It is of eminent significance that the partition function $Q$, as defined in the above manner, can be obtained from experimental heat capacity vs temperature curves by numerical integration of the equation

$$
Q=\exp \left[\int_{T_{0}}^{T}\left(\Delta H_{\text {excess }} / R T^{2}\right) d T\right]
$$

$T_{0}$ is the temperature at which all molecules exist in the initial state (intact three-dimensional structure) and the term $\Delta H_{\mathrm{e} \times \mathrm{c} \text { es s }}$ is the area under the heat capacity vs temperature curve at temperature $T$. Having the partition function at hand opens up all the possibilities of statistical thermodynamics.

Thermodynamically Defined, Cooperatively Unfolding Substates of Biological Macromolecules are frequently also Structurally Discrete Domains

An apparently simple example is furnished by unfolding a protein called $\lambda$ repressor. The heat capacity vs temperature curve displays two peaks that are very well separated on the temperature scale (Fig. 4). The narrowness of the peaks (actually the width at half height) immediately implies cooperative unfolding of structurally discrete parts of the protein, a result which is corroborated by non-thermodynamic techniques.

However, shape analysis of heat capacity curves allows us also to identify cooperatively unfolding domains in cases where overlapping transitions occur. In such situations, obviously care has to be taken not to over-interpret the results in structural or mechanistic terms without additional non-thermodynamic information ${ }^{6}$ ).

Fig. 3 - Typical heat capacity vs. temperature curve ('transition curve') of a cooperatively unfolding protein.

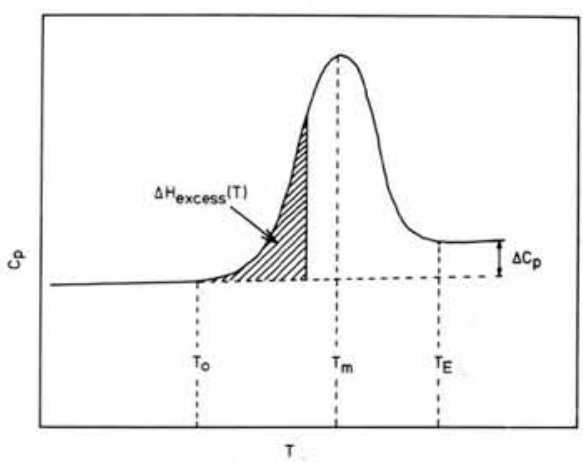




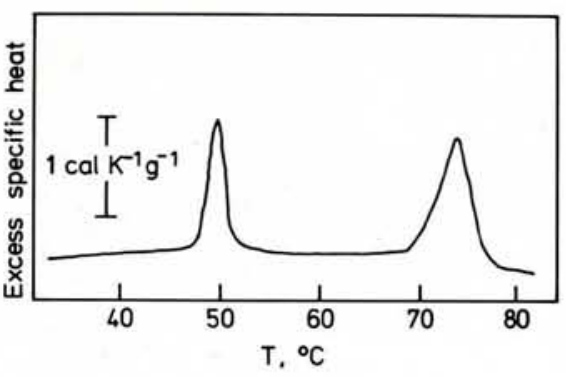

Fig. 4 - Heat capacity vs. temperature curve of a protein consisting of two structural domains of different stability ( $\lambda$-repressor, after Pabo et al., (1979) Proc. Natl. Acad. Sci. USA 76, 1608).

Nucleic Acid and Lipid Transitions are also Characterized by Cooperativity of Structural Domains

Small proteins having 50 to 100 amino acids unfold as one cooperative unit. However, small nucleic acids, such as transfer ribonucleic acids which consist of approximately 80 nucleotides organized in a well defined three-dimensional structure in solution, behave differently when exposed to increasing temperature. Clearly energetically separated intermediate states can be identified which coincide perfectly with structural domains of the molecule ${ }^{7}$ ). The reason why proteins must incorporate a larger number of structural units (amino acids) into a stable domain may result from the fact that the average interaction enthalpy, $\Delta H$, is approximately 30 $\mathrm{kJ} / \mathrm{mol}$ of nucleotide in nucleic acids, whereas it is only $1-3 \mathrm{~kJ} / \mathrm{mol}$ of amino acid in proteins.

We have seen that the thermodynamic parameters for proteins and nucleic acids relate to the difference between the random coil state of the macromolecule and the well defined three-dimensional structure.
The best understood thermodynamic quantities of lipid systems relate to temperature induced transitions between two lipid phases of a different degree of order within a two dimensional layer (Figs. 1b, c). Highly purified lipid systems exhibit phase transitions which appear to be of first order and which incorporate more than 1000 lipid molecules into one cooperative unit. It is obvious that these transitions offer a great challenge to theoreticians to elaborate treatments which match the experimental findings 8 ).

Microcalorimetry: Traditional Tool with Future Potential

The brief introduction into thermodynamic characterisation of biological systems has tried to demonstrate a) the usefulness of such a treatment and b) the outstanding suitability of high sensitivity scanning microcalorimetry in providing model free energy parameters and furthering our insight into the domain structure of biological macromolecules. Continuing research in this field will contribute to our understanding of the thermodynamic basis of biological systems, which, in contrast to chemistry and physics, has been unduly neglected in biology.

\section{REFERENCES}

1. Degiorgio V., Physics of Amphiphiles, Micelles and Microemulsions, Europhys. News 16 (1985) 6.

2. Sturtevant J.M., Physical Methods of Chemistry (eds. A. Weissberger and B.W. Rossiter) Vol. 1 Part V (Academic Press, NY) 1971, pp. 227-253.

3. Privalov P.L., Pure Appl. Chem. 52 (1980) 479-497.

4. Freire E. and Biltonen R., Biopolymers 17 (1978) 463

5. Gill S.J., Richey B., Bishop G. and Wyman J., Biophysical Chemistry 21 (1985) 1-14.

6. Privalov P.L., Adv. Prot. Chem. 35 (1982) 1-104.

7. Hinz H.J., Filimonov V.V. and Privalov P.L. Eur. J. Biochem. 72 (1977) 79-86.

8. Nagle J.F., Ann. Rev. Phys. Chem. 31 (1980) 157-195.

\section{6 \\ Hewlett-Packard Europhysics Prize}

The 1986 Hewlett-Packard Europhysics Prize has been awarded to:

Professor Ferenc Mezei

of Budapest, Grenoble and Berlin (West) for his invention and implementation of Neutron Spin Echo Spectroscopy (Europhys. News 16 (1985) 4).

\section{GRADUATE SUMMER COURSES ON COMPUTATIONAL PHYSICS}

\section{Lausanne, Beginning 1-10 September 1986}

The Computational Physics Group of EPS has been organizing for the past ten years a Summer School and Workshop on Computing Techniques in Physics. This school which takes place in Czechoslovakia every second year, elicits a good response especially from eastern countries.

In 1984 the Board of CPG decided to organize another Summer School in Lausanne, Switzerland, tending much more towards a graduate course. This Graduate Summer Course on Computational Physics will start in 1986 with its first course on "Finite Elements in Physics". During eight days (1-10 September 1986), 48 lectures will be given including a basic course and going on to practical applications.

The course will be published as a whole volume in Computer Physics Reports. For 1988 a second Graduate Summer Course is foreseen on "Numerical Methods for Parallel Computers".

Further information is available from the Secretariat of the course:

Mme C. Antille, Centre de Recherches en Physique des Plasmas - EPFL

21, av. des Bains, $\mathrm{CH} \cdot 1007$ LAUSANNE, Switzerland

EPS Divisions, Sections and Group

Astronomy and Astrophysics Division

Solar Physics Section

Atomic and Molecular Physics Division

Atomic Spectroscopy Section

Chemical Physics

Chemical Physics

Electronic and Ato
Molecular Physics

Computational Physics Group

Condensed Matter Division

Liquids Section

Low Temperature Physics Section

Macromolecular Physics

Magnetism

Magnetism
Metal Physics

Metal Physics

Semiconductors and Insu
Surfaces and Interfaces

High Energy \& Particle Physics Division

Interdiv. Group on Physics for Development

Nuclear Physics Division

Optics Division

Plasma Physics Division

Quantum Electronics Division
Europhysics News is the official journal of the European Physical Society which comprises 29 National Societies, Academies and Group, over 3500 Individual Members and 73 Associate Members. Governing bodies of EPS are the General Meeting, Council and an elected Executive Committee responsible for detailed policy. EPS promotes the collaboration of physicists throughout Europe, organising and harmonising conferences and publications, improving physics education, encouraging physics applications, awarding scholarships to sponsored schools in Erice. EPS publishes in addition to Europhysics News, Europhys. Conf. Abs., Eur. Ed. News, Europhys. Lett. (in partnership with national societies), Eur. J. Phys. (in partnership with The UK Inst. of Phys.). Individual Members receive Europhys. News free of charge (price to institutions: Sw.Fr. 90. la), rebates on the price of many publications and on conference fees. Annual EPS membership fee for Individual Members belonging to an EPS member society is: Sw.Fr. 44--; independent members: Sw.Fr. 132--; members of a Collaborating Society: Sw.Fr. 55.- (\$26).
Editor: E.N. Shaw

Editorial Board:

K. Appert, A. Baratoff, B. Jacrot,

G.R. Macleod, M. Mayor, J. Muller

Editorial and Advertising Office at the EPS Secretariat

Address: EUROPEAN PHYSICAL SOCIETY P.O. Box 69 , CH-1213 Petit-Lancy 2 Switzerland

Telephone: Geneva (22) 931130 Telex: $\mathbf{4 2 3} 455$ dema ch

Cables: europhys genève

Printed by: Pfirter frères se CH-1213 Petit-Lancy/Switzerland 